\title{
Restorative and therapeutic benefits of yoga in addiction recovery
}

\begin{abstract}
This cursory review identifies some of the current research linking Yogic Breathing and Mindfulness practices to alternative complementary approaches to addiction and rehabilitation programs.
\end{abstract}

Keywords: addiction, depression, anxiety, alcohol, treatment, yoga
Volume 3 Issue 5 - 2017

Mary Louisa Cappelli
Nevada State College, USA

Correspondence: Mary Louisa Cappelli, Participatory Action Research Network to improve the lives of indigenous women, Nevada State College, USA, Email mlcappelli@globalmother.org

Received: April 04, 2017 | Published: June 13, 2017
Abbreviations: GABA, gamma-aminobutyric acid; SNS, sympathetic nervous system; PNS, parasympathetic nervous system

\section{Introduction}

In recent years, a plethora of case studies have reported the therapeutic benefits of yoga, reinforcing previous suggestions that the combination of yoga with other treatments can reduce depression and anxiety and also assist in addiction recovery processes. ${ }^{1-4}$ Many of the case studies appear to be qualitative controlled trials.

\section{Discussion}

One such case study, conducted by O'Dell O Johnson, ${ }^{5}$ entitled "A Novel Approach to Alternative Integral Practices in African American Communities Challenged with Substance Abuse," demonstrates the helpfulness of integrating mindfulness breathing and yoga practices in conjunction with the 12-Step Program to support inner city drug users. The study determined that addiction relapse is a never-ending cycle because of the high disparity and disconnection of mind, body, and spirit. The implementation of mindfulness yoga training served to complement existing drug treatment methods by integrating holistic treatments in at-risk African American at the Hoover Treatment Center in Little Rock, Arkansas. Johnson, ${ }^{5}$ himself who is a recovering addict, piloted the program, which integrated meditation, yoga, and reiki and nutritional alternatives.

a. His findings support other key research that yoga practices can help addicts maintain sobriety. Johnson asserts that yoga forces the mind and body to work holistically together in unity and harmony.

b. Yoga appears to help moderate stress response systems by decreasing heart rate, lowering blood pressure and improving respiratory systems.

Other research confirms the findings that yoga has been found to increases levels of gamma-aminobutyric acid, (GABA) an amino acid which acts as an inhibitory neurotransmitter inhibiting nerve transmission in the brain. Individuals who suffer from depression and anxiety generally have low levels of GABA. Yoga practice has shown to improve these thalamic GABA levels. This is an important contribution to studies on alternative approaches to prescribing pharmacological agents in the treatment of mood disorders, anxiety, and depression, which are often linked to addiction. ${ }^{6,7}$
A University of Utah case study (2008) of 12 yoga practitioners, 14 fibromyalgia sufferers and 16 healthy non-yoga volunteers confirms these findings. The study concluded that both the volunteer and fibromyalgia groups felt pain at lower pressure levels than the yoga group, demonstrating that the yoga practitioners held the highest threshold for pain. This research highlights the therapeutic benefits of yoga as a stress, regulator as well as yoga's ability to reduce physical pain variables and emotional suffering.

In addition, studies have shown that regular yoga practice can, moreover, reduce cravings associated with addiction by reducing sympathetic nervous system (SNS) activity and activating parasympathetic nervous system (PNS) activity. ${ }^{8}$ One study concluded that Sudarshan Kriya Yoga, a method of controlled cyclical breathing, which moves from slow to rapid inhalation/exhalation, offers relief from alcohol dependency. ${ }^{1,9}$ At a detoxification program in Bangalore, India, 60 alcohol-addicted men were randomly enrolled in a threeweek Sudarshan Kriya yoga practice or a standard treatment program. Sudarshan Kriya yoga practioners reported a 75 percent reduction of depression and lower levels of cortisol and corticotropin cortisol. While the standard treatment group also reported a 60 percent drop in the standard treatment group, stress levels in the control group remained the same. The Bangalore case study suggests that yogic breathing is a beneficial treatment for addicts in the early stages of recovery.

Other research that a heated environment constitutes a further complementary therapy for addiction and depression. According to Maren Nyer, director of Yoga Research in MGH Depression and Clinical Research Program and professor of Psychiatry at Harvard Medical School, hot yoga may lead to the regulation and management of "physiological functions that often contribute to the reversal of a depressed state". ${ }^{10}$ Both Traditional and hot yoga practices combine mindfulness, postures, breathing, and meditation to focus attention to holistic alignment with the physical, emotional, and spiritual body. The mindfulness skills developed in yoga "can target clinically relevant measures of psychological, biological, and behavioral functioning, all of which are implicated in the pathophysiology of yoga". ${ }^{2}$ While there are many forms of yoga, Vinyasa, Hatha, Sstanga, Iyengar, to name a few, hot yoga takes place in a heated environment with temperatures averaging 105 Fahrenheit (40.5 Celsius). According to Maurizio Fava, MD and director of the MGH Depression Clinical and Research Program at Harvard Medical School," while research is still 
underway, the benefits of a "heated environment may enhance the antidepressant effects of this form of yoga". ${ }^{10-12}$

\section{Conclusion}

Scientific research on the therapeutic benefits of yoga for individuals suffering from depression, anxiety, and addiction is still in its early phases. More research needs to be undertaken to understand the potential use of yoga and its restorative abilities to help patients make beneficial steps toward sobriety, prevention, recovery and wellbeing in addiction treatment programs.

\section{Acknowledgements}

None.

\section{Conflict of interest}

The author declares no conflict of interest.

\section{References}

1. Brown RP, Gerbarg PL. Sudarshan Kriya yogic breathing in the treatment of stress, anxiety, and depression: part I-neurophysiologic model. $J$ Altern Complement Med. 2005;11(1):189-201.

2. Khanna S, Greeson JM. A narrative review of yoga and mindfulness as complementary therapies for addiction. Complement Ther Med. 2013;21(3):244-252.

3. Kirkwood G, Rampes H, Tuffrey V, et al. Yoga for Anxiety: A systematic review of the research evidence. Br J Sports Med. 2005;39(12):884-891.

4. Pilkington K, Kirkwood G, Rampes H, et al. Yoga for depression: The research evidence. $J$ Affect Disord. 2005;89(1-3):13-24.
5. O'Dell Johnson. A Novel Approach to Alternative Integral Practices in African American Communites Challenged with Substance Abuse. USA: NDN publication;

6. Streeter Chris C, Theodore H, Whitfield Liz Owen, et al. Effects of yoga versus walking on mood, anxiety, and brain GABA levels: a randomized controlled MRS study. J Altern Complement Med. 2010;16(11):11451152 .

7. Narasimhan L, Nagarathna R, Nagendra H. Effect of integrated yogic practices on positive and negative emotions in healthy adults. Int $J$ Yoga. 2011;4(1):13-29.

8. Telles S, Raghavendra BR, Naveen KV, et al. Changes in autonomic variables following two meditative states described in yoga texts. $J \mathrm{Al}$ tern Complement Med. 2013;19(1):35-42.

9. Janakiramaiah N, Gangadhar BN, Naga Venkatesha Murthy PJ, et al. Antidepressant efficacy of Sudarshan Kriya Yoga (SKY) in melancholia: a randomized comparison with electroconvulsive therapy (ECT) and imipramine. J Affect Disord. 2000;57(1-3):255-259.

10. Massachusetts General Hospital. Massachusetts General Hospital testing hot yoga for treatment of depression. 2013.

11. Khalsa SB. Yoga as a therapeutic intervention: a bibliometric analysis of published research studies. Indian J Physiol Pharmacol. 2004;48(3):269-285.

12. Streeter CC, Jensen JE, Perlmutter RM, et al. Yoga Asana Sessions Increase Brain GABA Levels: A Pilot Study. J Altern Complement Med. 2007;13(4):419-426. 